\title{
Stromal-cell and cancer-cell exosomes leading the metastatic exodus for the promised niche
}

\author{
Robert M Hoffman*1,2
}

\begin{abstract}
Exosomes are thought to play an important role in metastasis. Luga and colleagues have described the production of exosomes by stromal cells such as cancer-associated fibroblasts that are taken up by breast cancer cells and are then loaded with Wnt 11, which is associated with stimulation of the invasiveness and metastasis of the breast cancer cells. Previous studies have shown that exosomes produced by breast cancer cells are taken up by stromal fibroblasts and other stromal cells, suggesting that exosomes are agents of cross-talk between cancer and stromal cells to stimulate metastasis. Imaging of exosomes by labeling with fluorescent proteins will enlighten the process by which exosomes enhance metastasis, including premetastatic niche formation.
\end{abstract}

Exosomes are small (30 to $100 \mathrm{~nm}$ ) membrane vesicles that originate from the endosomal membrane compartment [1-3]. Exosomes contain mRNAs, miRNAs and proteins, and possibly other components as well $[1,4,5]$. Cancer cells release exosomes into the tumor microenvironment and peripheral blood $[1,6]$.

Do stromal cells in the tumor microenvironment also produce exosomes that stimulate cancer metastasis? We previously showed that cancer cell-associated stromal cells are necessary for metastasis to occur [7]. Luga and colleagues now report in a recent issue of Cell that stromal cells indeed produce exosomes and that fibroblast-secreted exosomes promote breast cancer cell (BCC) motility and metastasis by mobilizing autocrine Wnt11-induced planar cell polarity in the cancer cells [8]. Wnt11 is tethered to the fibroblast-derived exosomes within BCCs. Exosome stimulation of BCC invasion and metastasis was shown to be dependent on Wnt11

*Correspondence: Email: all@anticancer.com

'AntiCancer, Inc., 7917 Ostrow Street, San Diego, CA 92111, USA

Full list of author information is available at the end of the article produced in the BCCs. Cancer-associated fibroblast (CAF)-derived exosomes were internalized by $\mathrm{BCCs}$ and then loaded with Wnt11 in orthotopic mouse models of breast cancer. Luga and colleagues report that exosomes secreted from human breast CAFs stimulate BCC protrusive activity, motility, and metastasis. These properties are dependent on the exosome tetraspanin, Cd81, which is critical for exosome-stimulated BCC metastasis [8]. These are intriguing findings on how stromal cells promote metastasis via exosomes.

Previous suggestions indicated that recruited bone marrow progenitor cells generated a premetastatic niche to which the cancer cells metastasize [9-11]. Secreted factors in the bone marrow may be important to mobilize cells to form the premetastatic niche. Exosomes, derived from cancer cells, have recently been shown to have an important role in premetastatic niche formation [9-11]. However, Luga and colleagues do not suggest how stromal cell-derived exosomes play a role in the formation of a premetastatic niche [8].

Investigating premetastatic niche formation requires the possibility for dynamic imaging of exosomes in vivo. To image the fate of cancer cell-derived exosomes in orthotopic nude mouse models of breast cancer, we used GFP-tagged CD63, which is a general marker of exosomes [12]. BCCs were imaged to transfer their own exosomes to other cancer cells and normal lung tissue cells in culture. In orthotopic nude mouse models, BCCs secreted exosomes into the tumor microenvironment. Tumor-derived exosomes were incorporated into tumorassociated cells at a metastatic site, including CAFs (Figure 1), and in the circulation. These results suggest that tumor-derived exosomes may contribute to forming a niche to promote tumor growth and metastasis. Our results demonstrate the usefulness of GFP imaging to investigate the role of exosomes in cancer metastasis [12-15].

Both cancer cell-derived or stromal cell-derived exosomes are thus able to alter the tumor environment and may participate in forming a distant metastatic niche to promote metastasis. Dynamic imaging of exosomes derived from cancer or stromal cells in metastatic models may hence help us to understand the mechanism of 

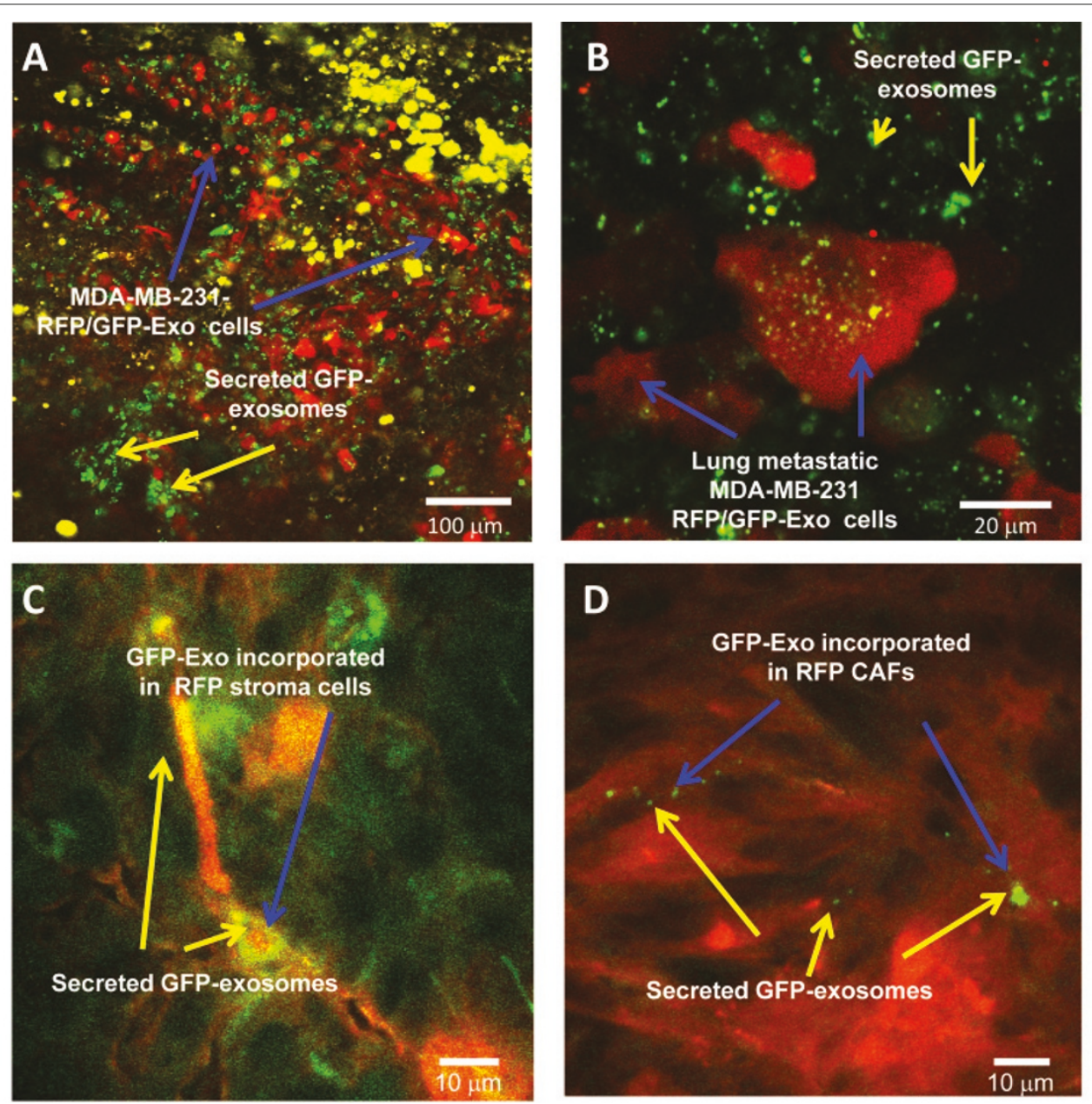

Figure 1. Cancer cells secrete exosomes into the tumor microenvironment in human MDA-MB-231 breast cancer orthotopic mouse models. The red fluorescent protein (RFP)-expressing MDA-MB-231-cells-produced exosomes which were labeled with a CD63-GFP fusion protein (MDA-MB-231-RFP/GFP-Exo). (A) MDA-MB-231-RFP/GFP-Exo cells secreted GFP exosomes in the primary tumor tissue. Blue arrows, MDA-MB-231RFP/GFP-Exo cells; yellow arrows, secreted GFP exosomes. Scale bar $=100 \mu \mathrm{m}$. (B) MDA-MB-231-RFP/GFP-Exo cells secreted GFP exosomes at the lung colonization site. Yellow arrows, secreted GFP exosomes; blue arrows, lung metastatic MDA-MB-231-RFP/GFP-Exo cells. Scale bar $=20 \mu \mathrm{m}$. (C) Blue arrows, GFP-Exo incorporated in RFP stroma cells; yellow arrows, secreted GFP exosomes. Scale bar $=10 \mu \mathrm{m}$. (D) Blue arrows, GFP-Exo incorporated in RFP cancer-associated fibroblasts (CAFs); yellow arrows, secreted GFP exosomes. Scale bar $=10 \mu \mathrm{m}$ [12].

cancer metastasis. Imaging of exosomes may also be useful to predict the location of future metastasis in real time.

This viewpoint demonstrates the importance of exosome cross-talk between cancer cells and stromal cells. Luga and colleagues demonstrate the production of exosomes by stromal cells such as CAFs that are taken up by BCCs, which in turn promote their invasive and metastatic activity [8]. Suetsugu and colleagues demonstrate production of exosomes by BCCs that are taken up by CAFs [10], the reciprocal of what was observed by Luga and colleagues [8]. With the use of fluorescentprotein in vivo imaging [13-15], further understanding of exosomes and their relationship to metastasis, including niche formation, will surely be enlightened.

\section{Abbreviations}

BCC, breast cancer cell; CAF, cancer-associated fibroblast; GFP, green fluorescent protein; miRNA, microRNA.

\section{Competing interests}

The author declares that he has no competing interests.

\section{Author details}

'AntiCancer, Inc., 7917 Ostrow Street, San Diego, CA 92111, USA. ${ }^{2}$ Department of Surgery, University of California San Diego, 7917 Ostrow Street, San Diego, CA 92111, USA.

\section{Published: 18 June 2013}

\section{References}

1. Ratajczak J, Wysoczynski M, Hayek F, Janowska-Wieczorek A, Ratajczak MZ: Membrane-derived microvesicles: important and underappreciated mediators of cell-to-cell communication. Leukemia 2006, 20:1487-1495.

2. Simons M, Raposo G: Exosomes - vesicular carriers for intercellular 
communication. Curr Opin Cell Biol 2009, 21:575-581.

3. Théry C, Ostrowski M, Segura E: Membrane vesicles as conveyors of immune responses. Nat Rev Immunol 2009, 9:581-593.

4. Skog J, Würdinger T, van Rijn S, Meijer DH, Gainche L, Sena-Esteves M, Curry Jr WT, Carter BS, Krichevsky AM, Breakefield XO: Glioblastoma microvesicles transport RNA and proteins that promote tumour growth and provide diagnostic biomarkers. Nat Cell Biol 2008, 10:1470-1476

5. Valadi H, Ekström K, Bossios A, Sjöstrand M, Lee JJ, Lötvall JO: Exosome mediated transfer of mRNAs and microRNAs is a novel mechanism of genetic exchange between cells. Nat Cell Biol 2007, 9:654-659.

6. Rabinowits G, Gerçel-Taylor C, Day JM, Taylor DD, Kloecker GH: Exosomal microRNA: a diagnostic marker for lung cancer. Clin Lung Cancer 2009, 10:42-46.

7. Bouvet $M$, Tsuji $K$, Yang M, Jiang P, Moossa AR, Hoffman RM: In vivo colorcoded imaging of the interaction of colon cancer cells and splenocytes in the formation of liver metastases. Cancer Res 2006, 66:11293-11297.

8. Luga V, Zhang L, Viloria-Petit AM, Ogunjimi AA, Inanlou MR, Chiu E, Buchanan M, Hosein AN, Basik M, Wrana JL: Exosomes mediate stromal mobilization of autocrine Wnt-PCP signaling in breast cancer cell migration. Cell 2012, 151:1542-1556.

9. Peinado H, Lavotshkin S, Lyden D: The secreted factors responsible for pre-metastatic niche formation: old sayings and new thoughts. Semin Cancer Biol 2011, 21:139-146.
10. Jung T, Castellana D, Klingbeil P, Cuesta Hernández I, Vitacolonna M, Orlicky DJ, Roffler SR, Brodt P, Zöller M: CD44v6 dependence of premetastatic niche preparation by exosomes. Neoplasia 2009, 11:1093-1105.

11. Hood JL, San RS, Wickline SA: Exosomes released by melanoma cells prepare sentinel lymph nodes for tumor metastasis. Cancer Res 2011, 71:3792-3801.

12. Suetsugu A, Honma K, Saji S, Moriwaki H, Ochiya T, Hoffman RM: Imaging exosome transfer from breast cancer cells to stroma at metastatic sites in orthotopic nude mouse models. Adv Drug Deliv Rev 2013, 65:383-390.

13. Hoffman RM: The multiple uses of fluorescent proteins to visualize cancer in vivo. Nat Rev Cancer 2005, 5:796-806.

14. Hoffman RM, Yang M: Subcellular imaging in the live mouse. Nat Protoc 2006, 1:775-782

15. Hoffman RM, Yang M: Color-coded fluorescence imaging of tumor-host interactions. Nat Protoc 2006, 1:928-935.

doi:10.1186/bcr3426

Cite this article as: Hoffman RM: Stromal-cell and cancer-cell exosomes

leading the metastatic exodus for the promised niche. Breast Cancer

Research 2013, 15:310. 